\title{
Analytical measurement method for situation elements' dynamic characteristics
}

\author{
Huiyun Zhao ${ }^{1}$, Dongge Zhang ${ }^{1, *}$, Hui Meng ${ }^{2}$, and Jiuyang Tao ${ }^{1,3}$ \\ 1. College of Command and Control System, Army Engineering University, Nanjing 210007, China; \\ 2. Military Representative Office of Taiyuan Railway Bureau, Taiyuan 030013, China; \\ 3. Department of Information Operation \& Command Training, National Defense University, Beijing 100091, China
}

\begin{abstract}
This paper gives an analysis of the dynamic characteristics of situation elements (SEs) in situation awareness (SA) research. The purpose of the discussion is to understand the factors that influence SA and to help in designing the training systems to improve operators' SA. The status function of SEs is defined and the derivative of the function represents trends of the status of SEs at each moment. Then, Fourier transform (FT) is used to give the frequency-domain function in terms of the time-domain status function. In frequency domain, the bandwidth of the status function is used as a criterion to characterize the notion of "fast" and "slow" of the change of SE's status, which represents the dynamic characteristic of SEs. The criterion constitutes the first analytical measurement of the dynamic characteristic of SEs, which is one of the important factors that influence the SA process.
\end{abstract}

Keywords: situation awareness (SA), furious transform (FT), situation elements (SEs), status value.

DOI: $10.21629 / J S E E .2017 .06 .10$

\section{Introduction}

This paper proposes a discussion on the dynamic characteristic of situation elements (SEs) in situation awareness (SA) research. SA is an important issue which needs to be addressed in many disciplines, such as humancontrolled and human-monitored mobile communication networks [1], patient care [2], information security risk management [3], social networks, intelligent transportation systems [4], and operational battlefield command and control [5] and so on [6-19]. Many definitions of SA are found in the literature, and the most commonly accepted definition is "the perception of the elements in the environment within a volume of time and space, the comprehension of their meaning, and the projection of their status

Manuscript received September 01, 2016.

*Corresponding author.

This work was supported by the National Natural Science Foundation of China (61174198) and the PLA Military Graduate Students Foundation (2011JY002-163). in the near future," which was proposed by Endsley in 1995 [1,20]. In addition to individual SA, team SA and distributes SA are also highly concerned by researchers [21 25]. According to the definitions and related research, SA of an operator is influenced by plenty of factors such as attention, stress [26], working memory, information availability and goals [27-32]. The above factors are concluded from the perspective of the operator. In fact, the dynamic characteristic of the SE that the operator needs to perceive and understand also influences the SA of an operator [33,34].

The dynamic characteristic can be used to determine which SE changes fast and which changes slowly. If an SE changes slowly, it is easy for operators to perceive, understand, and even to predict the status of this SE in the near future. However, for an SE that changes fast, getting a high-level SA is difficult for operators. Take commanders on the battlefield as an example: the position of a missile changes so quickly that the commander hardly knows the specific position of the missile even with the help of the advanced command and control information system. However, if the target is a tank, it is easier for the same commander to predict the position of the tank. Therefore, the study of the dynamic characteristic of SEs is useful for SA estimation of certain operators and system designing.

However, the existing research rarely involves the dynamic characteristic of SEs since the types of SEs are extremely varied and their dynamic characters are different from one another. Our research proposes that the status of an SE can be represented as a function named status function of the SE, the derivative of which describes the trends of the SE at each moment. Fourier transform (FT) is used to analyze the status value function in the frequency-domain, and the bandwidth of the status value function is defined to 
evaluate the dynamic characteristic of the SE.

\section{Status function of the SE}

Characteristics of an SE include two parts: status and the dynamic characteristic. The description of status of an SE is needed to analyze the dynamic characteristic of the SE. Therefore, some basic definitions about the status of the SE are discussed in this section.

Operators usually obtain the status of SEs from information systems. In systems like military command and control information systems, sensors take "snapshots" of the battlefield environment at regular intervals to get status value of SEs, which will then be displayed to operators through the information system. Therefore, operators get the status of SEs at each snapshot. In general, we can fit the status of SEs by mathematical functions.

Definition 1 Status function $f$ : The changing status of situation element $A$ is represented by the function $f_{A}$, and $f_{A}(t)$ is the status of $A$ at time $t$.

A snapshot is a sampling of status of SEs. To help operators accurately understand changes of SEs, sensor systems usually gather information periodically. Thus, operators generally get situation information (SI) at a fixed frequency.

Definition 2 Sampling frequency $R$ : The time between two snapshots is $\Delta t$, which means the sampling period is $\Delta t$; therefore, the sampling frequency $R=\frac{1}{\Delta t}$.

The changing rates of the status of various SEs are different from one another. Therefore, different sampling frequencies are used to meet the actual demand. High sampling frequency can help the operator know the current situation when the status of an SE changes fast or the mission of the operator is with real-time requirements, and vice versa. For instance, the position of a missile is an SE that changes rapidly for military commanders, therefore, it is necessary to set a high sampling frequency in the information system for commanders to fully know the position of the missile.

Since the derivative of a function describes the trends of the function in each value of the independent variable in mathematics, we define the trend of status at a particular moment based on the definition of the status function of the SE as follows:

Definition 3 Status trends function $f^{\prime}$ : If the status of situation element $A$ is expressed by $f_{A}$, then the status trends at time $t$ is $f_{A}^{\prime}(t)$, where $f_{A}^{\prime}$ is derivative of $f_{A}$.

The sign of $f_{A}^{\prime}(t)$ shows whether the status of $A$ is increasing or decreasing and the value of $f_{A}^{\prime}(t)$ reflects the intensity of the change.

Take the flight altitude of aircraft A as an example. $f_{\text {alt_A } A}(t)$ describes the flight altitude of aircraft $\mathrm{A}$ in an hour, and $f_{\text {alt_A }}^{\prime}(t)$, the derivative of $f_{\text {alt } \_A}(t)$, reflects the trend of the flight altitude at each moment. Fig. 1 shows $f_{\text {alt } \_} A(t)$ and $f_{\text {alt } \_A}^{\prime}(t)$. When $t=0.8 \mathrm{~h}$, the flight altitude is $f_{\text {alt_A } A}(0.8)=10 \mathrm{~km}$, and $f_{\text {alt_ } A}^{\prime}(0.8)=0.23$. The flight altitude of aircraft A rises sharply.

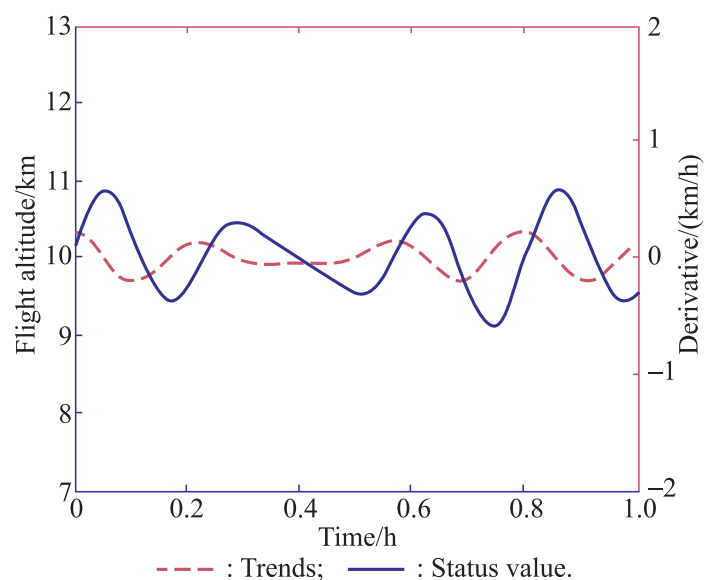

Fig. 1 Current status information and trends information (derivative) of aircraft $A$

\section{Dynamic characteristics of the $\mathrm{SE}$}

Although the status trends function represents variation of the status at each moment, whether the SE changes fast or slowly as a whole is still unknown. For example, curves in Fig. 2 represent the flight altitude of two aircrafts during a task, and we are unable to determine which of them changes fast through the curves. Set the status function of the two flight altitudes as $h_{1}(t)$ and $h_{2}(t)$, and the status trends function $h_{1}^{\prime}(t)$ and $h_{2}^{\prime}(t)$ show the variation of the flight altitude at each moment in Fig. 3. Comparing the curves of $h_{1}^{\prime}(t)$ and $h_{2}^{\prime}(t)$ in Fig. 3, we cannot determine which aircraft's flight altitude changes faster.

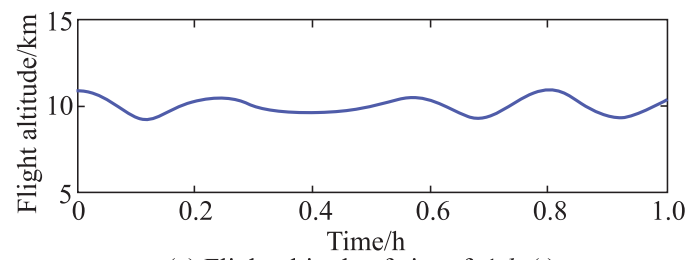

(a) Flight altitude of aircraft $1 h_{1}(t)$

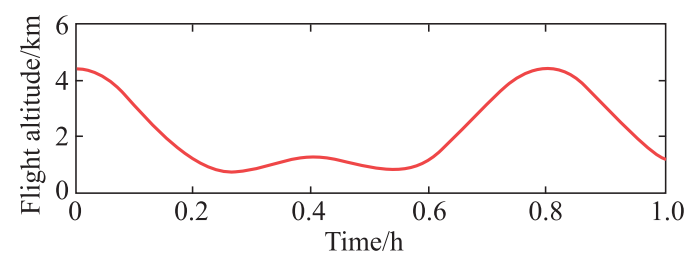

(b) Flight altitude of aircraft $2 h_{2}(t)$

Fig. 2 Flight altitude $h(t)$ of the aircraft 


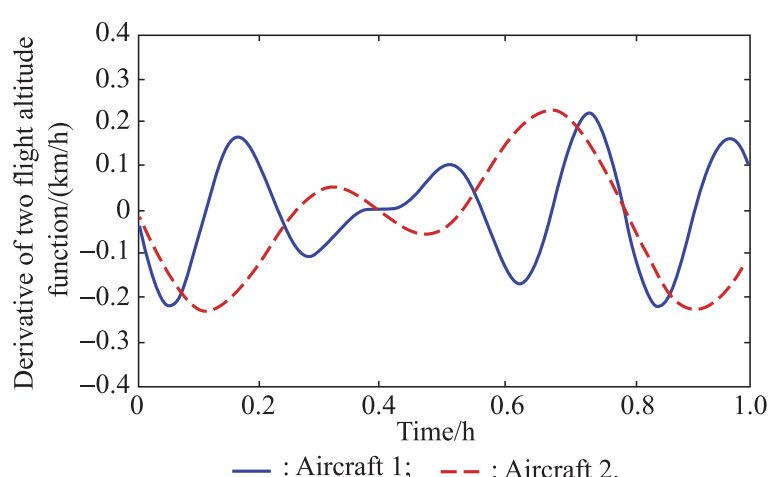

Fig. 3 Derivative $h^{\prime}(t)$ of flight altitude $h(t)$

In this section, FT is used to transform the status function of SEs from the time-domain into the frequencydomain, and then the bandwidth of the status function in the frequency-domain is defined as a criterion to characterize the notion of "fast" and "slow" of the change of SEs.

\subsection{Status function of the SA in frequency-domain}

In the signal processing, time and frequency describe the same signal from two different aspects [35]. FT is a formula that gives the frequency-domain function in terms of the time-domain function. FT takes sine and cosine signals as the basic unit, and then arbitrary signals can be described as sums of sine and cosine signals in Fig. 4. Signals are functions, so we can also use FT to transform the status value function of the SE from the time-domain to the frequency-domain.

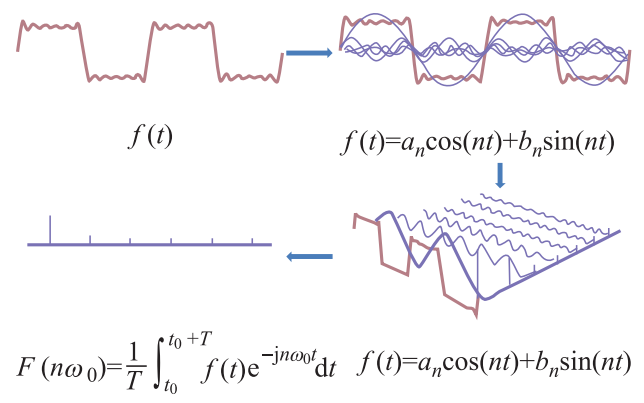

Fig. 4 Schematic diagram of FT

\subsubsection{Fourier series of periodic status value function}

Fourier series (FS) can be used to periodically describe the changed SE. Suppose the period of function $f(t)$ is $T$, if $f(t)$ satisfies Dirichlet conditions, which include three things [33]:

(i) in any finite interval, $f(t)$ is continuous at all but a finite number of points;

(ii) in any finite interval, $f(t)$ is of bounded variation, which means that there are no more than a finite number of maxima or minima; (iii) $f(t)$ is absolutely integrable,

$$
\int_{t_{0}}^{t_{0}+T}|f(t)| \mathrm{d} t<\infty
$$

then, $f(t)$ can be described as a weighted sum of complex exponentials.

$$
\begin{gathered}
f(t)=a_{0}+a_{1} \cos \omega_{0} t+a_{2} \cos 2 \omega_{0} t+\cdots= \\
b_{1} \sin \omega_{0} t+b_{2} \sin 2 \omega_{0} t+\cdots= \\
a_{0}+\sum_{n=1}^{\infty}\left[a_{n} \cos \left(n \omega_{0} t\right)+b_{n} \sin \left(n \omega_{0} t\right)\right]= \\
c_{0}+\sum_{n=1}^{\infty} c_{n} \cos \left(n \omega_{0} t+\phi_{n}\right)
\end{gathered}
$$

For (2):

$$
\begin{gathered}
a_{0}=c_{0}=\frac{1}{T} \int_{t_{0}}^{t_{0}+T} f(t) \mathrm{d} t \\
a_{n}=\frac{2}{T} \int_{t_{0}}^{t_{0}+T} f(t) \cos \left(n \omega_{0} t\right) \mathrm{d} t \\
b_{n}=\frac{2}{T} \int_{t_{0}}^{t_{0}+T} f(t) \sin \left(n \omega_{0} t\right) \mathrm{d} t \\
c_{n}=\sqrt{a_{n}^{2}+b_{n}^{2}} \\
\phi_{n}=-\arctan \frac{b_{n}}{a_{n}}
\end{gathered}
$$

where $\omega_{0}$ is the fundamental frequency in radians per second, $\omega_{0}=\frac{2 \pi}{T}$, and $t_{0}=-\frac{T}{2}$ [35].

According to Definition 1, the status value function about situation element $A$ is $f_{A}(t)$. When the sampling period $\Delta t$ of an operator is small enough, in which case the sampling frequency $R \rightarrow \infty$, then $f_{A}(t)$ can be regarded as continuous. Additionally, since the status value of the SE describes some physical quantities, it should be bounded. Therefore, $f_{A}(t)$ satisfies Dirichlet conditions, so that $f_{A}(t)$ can be represented by (2), and the frequency of the components are integral multiples of $\omega_{0}$.

Take the flight altitudes of aircraft 1 and aircraft 2 in Fig. 2 as an example. The status value functions $h_{1}(t)$ and $h_{2}(t)$ are periodic functions where

$$
\begin{gathered}
h_{1}(t)=10+\frac{1}{3 \pi} \cos (2.5 \pi t)+\frac{5}{3 \pi} \cos (7.5 \pi t)+\frac{1}{\pi} \cos (10 \pi t) \\
h_{2}(t)=2+\frac{5}{\pi} \cos (2.5 \pi t)+\frac{4}{3 \pi} \cos (5 \pi t) .
\end{gathered}
$$

The unit of the flight altitude is $\mathrm{km}$, and the period of both is $T=0.8 \mathrm{~h}$. After the FT, the magnitude of the FS coefficients of $h_{1}(t)$ and $h_{2}(t)$ are discrete as can be seen in Fig. 5 and Fig. 6, and the magnitude of components is different from each other. 


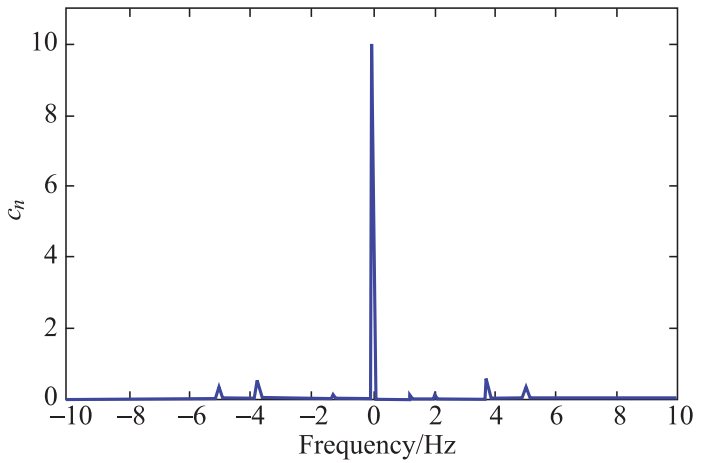

Fig. 5 Magnitude of the FS coefficients of aircraft 1's flight altitude

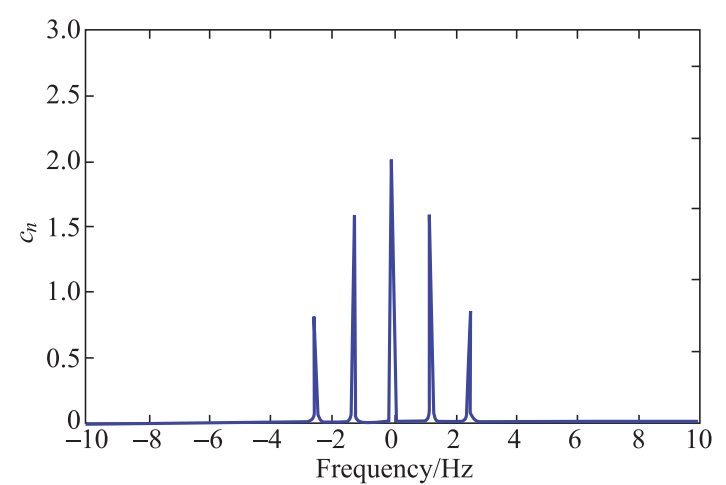

Fig. 6 Magnitude of the FS coefficients of aircraft 2's flight altitude

\subsubsection{FT of aperiodic status value function}

For aperiodic status value function, FT gives the frequency-domain function in terms of the time-domain function. Suppose $f(t)$ is an aperiodic function, if $f(t)$ is absolutely integrable, then it has FT.

$$
\begin{gathered}
F(\omega)=\int_{-\infty}^{\infty} f(t) \mathrm{e}^{-\mathrm{j} \omega t} \mathrm{~d} t \\
f(t)=\frac{1}{2 \pi} \int_{-\infty}^{\infty} F(\mathrm{j} \omega) \mathrm{e}^{\mathrm{j} \omega t} \mathrm{~d} \omega
\end{gathered}
$$

Equation (10) is the FT and (11) is the inverse FT. $F(\mathrm{j} \omega)$ in (10) and (11) can also be written in the form of trigonometric functions:

$$
f(t)=\frac{1}{2 \pi} \int_{-\infty}^{\infty}|F(\omega)| \cos (\omega t+\varphi(\omega)) \mathrm{d} \omega .
$$

Equation (10) shows that an aperiodic function can also be expressed as a sum of trigonometric functions. The period $T$ of an aperiodic function is infinity and the fundamental frequency is infinitesimal. Therefore, frequencies of the sinusoidal functions range from 0 to infinity. For the arbitrary signal whose energy is limited, amplitude
$\frac{F(\mathrm{j} \omega)}{2 \pi} \mathrm{d} \omega$ of the components at different frequencies is infinitesimal. $|F(\mathrm{j} \omega)|$ is the magnitude of the FT of $f(t)$, and it reflects the relative size of amplitudes of those trigonometric functions.

The status value function is a physical quantity, thus, it is finite and continuous. As a result, FT can be used to transform the aperiodic status value function.

Fig. 7 shows the distance between two tanks and their destination in 10 minutes. The moment we start to sample the distance is the initial moment. Suppose the distance during this period is in line with the exponential function, distance between tank 1 and its destination is $d_{1}(t)$ and distance between tank 2 and its destination is $d_{2}(t)$. The unit of the distance is $\mathrm{km}$.

$$
\begin{aligned}
& d_{1}(t)=60 \mathrm{e}^{-0.1 t} \\
& d_{2}(t)=60 \mathrm{e}^{-0.5 t}
\end{aligned}
$$

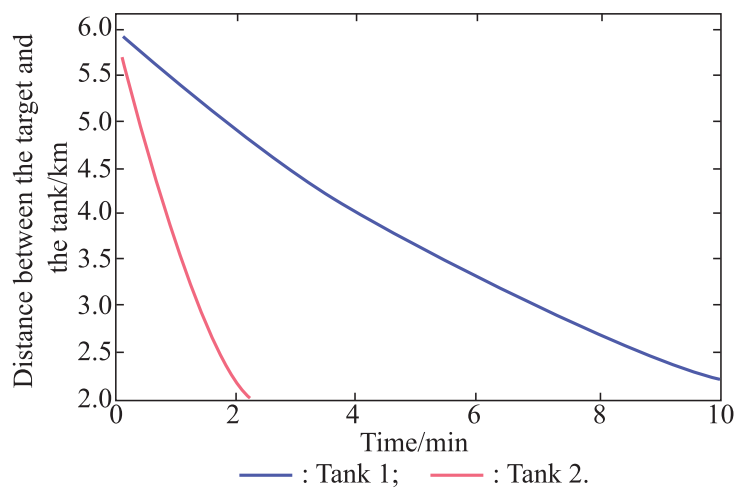

Fig. 7 Distance between two tanks and their destinations

According to the FT of the aperiodic status value function, the magnitudes of the FT of $d_{1}(t)$ and $d_{2}(t)$ are shown in Fig. 8 and Fig. 9.

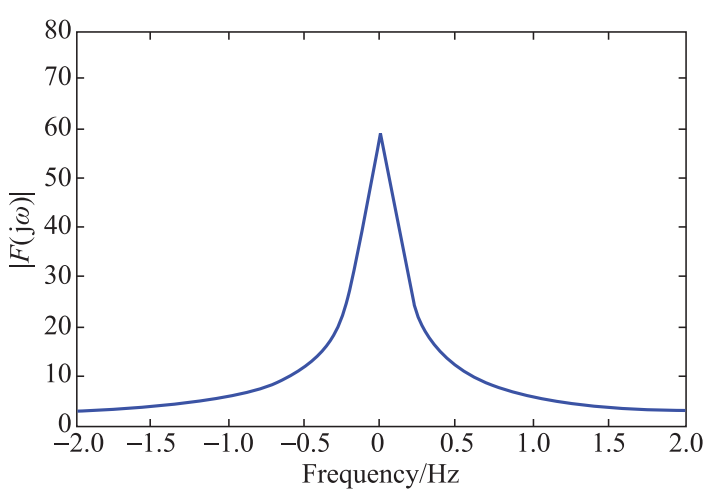

Fig. 8 Magnitude of FT of $d_{1}(t)$ 


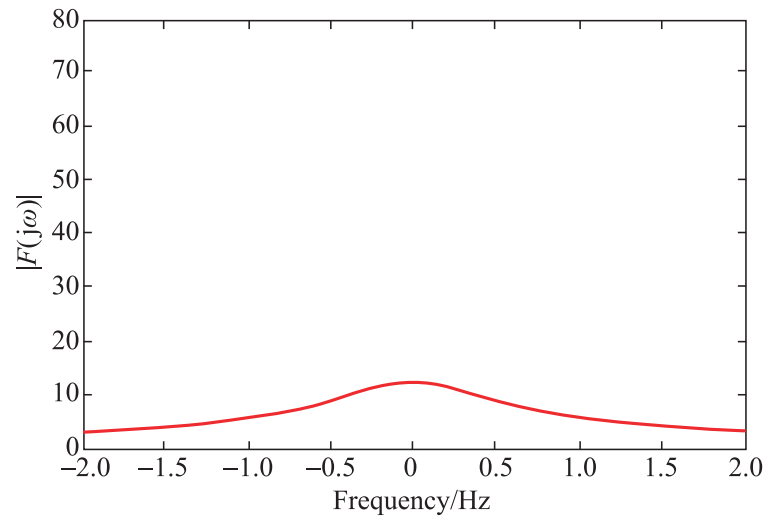

Fig. 9 Magnitude of FT of $d_{2}(t)$

\subsection{Analysis of dynamic characteristics of the SE}

In this section, we discuss how to use the bandwidth to access the dynamic characteristics of the SE.

In the frequency-domain, we use the bandwidth of the status value function of an SE to analyze the dynamic characteristics of the SE. The term bandwidth is applied to both signals and systems. There are many different definitions of the bandwidth. Some definitions are based on the signal power and others on the decay of the signal magnitude. In our research, $f(t)$, which is the status value function of the SE, can be represented as the sum of trigonometric functions. Since these trigonometric functions have different amplitudes, each trigonometric function has a different influence on $f(t)$. The larger the amplitude of a sine or cosine signal is, the greater it effects $f(t)$. Therefore, we use the definition of the bandwidth based on the decay of the signal amplitude to describe the effects of different coefficients of $f(t)$.

Definition 4 Bandwidth of status value function: the bandwidth is the distance between 0 and the point where the signal is $1 / \sqrt{2}$ of the maximum signal amplitude in the frequency-domain.

Set $F(\mathrm{j} \omega)$ as the result of FT of $f(t)$, then the magnitude of $F(\mathrm{j} \omega)$ is $|F(\mathrm{j} \omega)| .|F(\mathrm{j} \omega)|$ is maximal when $\omega=0$. The maximum value is expressed as $|F(0)|$. If $\omega_{c}\left(\omega_{c}>0\right)$ satisfies $\left|F\left(\mathrm{j} \omega_{c}\right)\right|=\frac{1}{\sqrt{2}}|F(0)|, \omega_{c}$ is the bandwidth of the status value function $f(t)$. For the periodic status value function $f(t)$ whose amplitude spectrum is discrete, $\omega_{c}$ is the bandwidth of $f(t)$ if $\omega_{c}$ satisfies

$$
\begin{gathered}
{\left[\left|F\left(\mathrm{j} \omega_{c}\right)\right| \geqslant \frac{1}{\sqrt{2}}|F(0)|\right]} \\
{\left[\left|F\left(\mathrm{j} \omega_{c}\right)\right|-\frac{1}{\sqrt{2}}|F(0)| \leqslant|F(\mathrm{j} \omega)|-\frac{1}{\sqrt{2}}|F(0)|\right]} \\
0 \leqslant \omega \leqslant \omega_{c} .
\end{gathered}
$$

According to the definition of the bandwidth of the status value function $f(t)$, when $\omega \leqslant \omega_{c}$, the amplitude of corresponding components of $f(t)$ in frequency domain is large enough to influence $f(t)$; and for those components whose frequency $\omega$ is less than $\omega_{c}$, their amplitude is less than $1 / \sqrt{2}$ of the maximum amplitude and their influence on $f(t)$ is too small to consider.

Therefore, if the bandwidth of the status value of the SE is large, the influence of high-frequency components in $f(t)$ is greater than other components. High frequency implies rapid change, therefore, the SE that has a large bandwidth changes fast. Taking the SE in Fig. 8 as an example, the distance between tank 1 and its destination is $d_{1}(t)$, and according to the magnitude of $d_{1}(t)$, maximum amplitude is $|F(0)|=59.30$. Since $\left|F\left(j \omega_{c 1}\right)\right|=|F(0)| / \sqrt{2}=$ 41.94 , then the bandwidth of $d_{1}(t)$ is $\omega_{c 1}=0.097 \mathrm{~Hz}$. In the same way, the bandwidth of $d_{2}(t)$ is $\omega_{c 2}=0.5 \mathrm{~Hz}$. Schematic diagrams of the bandwidth of $d_{1}(t)$ and $d_{2}(t)$ are shown in Fig. 10 and Fig. 11. Since $\omega_{c 2}>\omega_{c 1}$, the distance between tank 2 and its destination changes faster.

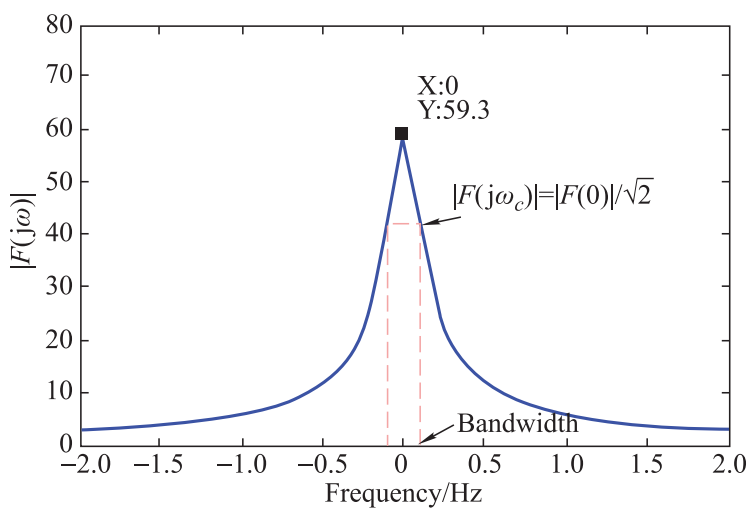

Fig. 10 Schematic diagram of the bandwidth of $d_{1}(t)$

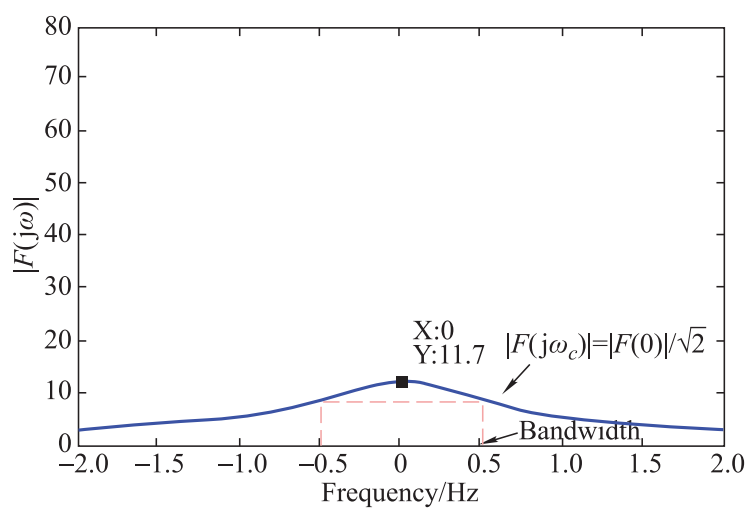

Fig. 11 Schematic diagram of the bandwidth of $d_{2}(t)$

Now, we can analyze the dynamic characteristics of $h_{1}(t)$ and $h_{2}(t)$ in Fig. 2. As mentioned at the beginning in Section 3, the derivation cannot be used to access the 
dynamic characteristic in this example because it is difficult to compare the value of $h_{1}^{\prime}(t)$ and $h_{2}^{\prime}(t)$ at different time. However, the proposed method does not need to consider this problem. For $h_{1}(t)$, the maximum amplitude in the frequency-domain is $|F(0)|=10,|F(0)| / \sqrt{2}=$ 7.07 , then according to (15), the bandwidth of $h_{1}(t)$ is $\omega_{c 1}=0 \mathrm{~Hz}$. And for $h_{2}(t)$, the maximum amplitude in the frequency-domain is $|F(0)|=2,|F(0)| / \sqrt{2}=1.41$, and the bandwidth of $h_{2}(t)$ is $\omega_{c 1}=1.25 \mathrm{~Hz}$. Since $\omega_{c 1}<\omega_{c 2}$, the flight altitude of aircraft 1 changes more slowly than the flight altitude of aircraft 2 . In practice, although the flight altitude of aircraft 1 fluctuates rapidly up and down, the total altitude of aircraft 1 stabilizes at $10 \mathrm{~km}$, which implies that this small rapid fluctuation does not have very big impact on the whole. However, for aircraft 2, though the number of the fluctuations is low, the range of these fluctuations is large and this leads to the result that $h_{2}(t)$ changes faster than $h_{1}(t)$.

The above example fully proves that the frequency domain analysis method proposed in this paper can effectively determine the dynamic characteristics of the SE.

\section{Conclusions}

A method based on FT and bandwidth of the status value function of the SE defined in this paper is used to analyze dynamic characteristics of the SE. Our results have implications for researches such as battlefield SA and intelligence analysis.

Nonetheless, since the method is in line with some hypotheses, and the definition of the status value function of the SE is limited by some conditions, more work is needed to extend the application fields of the method.

\section{References}

[1] M. M. Kokar, M. R. Endsley. Situation awareness and cognitive modeling. IEEE Intelligent Systems, 2012, 27(3): 91-96.

[2] C. M. Schulz, M. R. Endsley, E. F. Kochs, et al. Situation awareness in anesthesia concept and research. Anesthesiology, 2013, 118(3): 729-742.

[3] J. Webb, A. Ahmad, S. B.Maynard, et al. A situation awareness model for information security risk management. Computers \& Security, 2014, 44(7): 1-15.

[4] C. A. Bolstad, M. R. Endsley, A. M. Costello, et al. Evaluation of computer-based situation awareness training for general aviation pilots. The International Journal of Aviation Psychology, 2010, 20(3): 1-26.

[5] J. Głowacka, J. Krygier, M. Amanowicz. A trust-based situation awareness system for military applications of the internet of things. Proc. of the 2nd IEEE World Forum on the Internet of Things, 2015: 490-495.

[6] M. M. Lenox, E. S. Connors, M. R. Endsley. A baseline evaluation of situation awareness for electric power system operation supervisors. Proc. of the Human Factors and Ergonomics Society Annual Meeting, 2011: 2044-2048.
[7] M. Deasi, M. Medvedev, M. Vazquez, et al. Influence of situation awareness on control allocation for remote robots. Proc. of the IEEE International Conference on the Technologies for Practical Robot Applications, 2013: 1-6.

[8] V. Tardan, L. Bourgeon, F. Darses. How do team leaders communicate to develop situation awareness? an exploratory study in a nuclear submarine simulator. Proc. of the European Conference on Cognitive Ergonomics, 2016: 1-4.

[9] M. Zuparic, M. Jobst, I. Macleod. Situation awareness and campaign assessments. Journal of the Operational Research Society, 2017, 68(1): 1-13.

[10] F. P. Pai, L. J. Yang, Y. C. Chung. Multi-layer ontology based information fusion for situation awareness. Applied Intelligence, 2017, 46(2): 1-23.

[11] M. R. Endsley. Final reflections situation awareness models and measures. Journal of Cognitive Engineering and Decision Making, 2015, 9(1), $101-111$.

[12] D. Gawlick. Mastering situation awareness: the next frontier. Proc. of the 7th Biennial Conference on Innovative Data Systems Research, 2015.

[13] E. Blasch, D. Shen, K. D. Pham, et al. Review of game theory applications for situation awareness. Proc. of SPIE 9469, Sensors and Systems for Space Applications VIII, 94690I, 2015: $1-10$.

[14] S. L. Schwartz. A comparison between functional and traditional interface displays in support of console operator performance and workload. http://etd.lsu.edu/docs/available/etd09292016-190404/.

[15] E. Blasch, D. Shen, K. D. Pham, et al. A task analysis toward characterizing cyber-cognitive situation awareness (CCSA) in cyber defense analyst. Proc. of the IEEE International MultiDisciplinary Conference on the Cognitive Methods in Situation Awareness and Decision Suppor, 2016: 14-20.

[16] M. R. Endsley. Situation awareness: operationally necessary and scientifically grounded. Cognition, Technology \& Work, 2015, 17(2): $163-167$.

[17] P. M. Salmon, M. G. Lenne, G. H. Walker, et al. Exploring schema-driven differences in situation awareness between road users: an on-road study of driver, cyclist and motorcyclist situation awareness. Ergonomics, 2014, 57(2): 191-209.

[18] K. S. O’Brien, D. O'Hare. Situation awareness ability and cognitive skills training in a complex real-world task. Ergonomics, 2007, 50(7): $1064-1091$.

[19] S. Loft, V. Bowden, J. Braithwaite, et al. Situation awareness measures for simulated submarine track management. Human Factors, 2015, 57(2): 298-310.

[20] M. R. Endsley. Situation awareness misconceptions and misunderstandings. Journal of Cognitive Engineering and Decision Making, 2015, 9(1): 4-32.

[21] E. Salas, C. Prince, D. P. Baker, et al. Situation awareness in team performance: implications for measurement and training. Human Factors, 1995, 37(1): $123-136$.

[22] J. Kitchin, C. Baber. A comparison of shared and distributed situation awareness in teams through the use of agent-based modeling. Theoretical Issues in Ergonomics Science, 2016, 17(1): $8-41$.

[23] J. Lundberg. Situation awareness systems, states and processes: a holistic framework. Theoretical Issues in Ergonomics Science, 2015, 16(5): 447-473.

[24] A. A. Cain, T. Edwards, D. A. Schuster. Quantitative measure 
for shared and complementary situation awareness. Proc. of the Human Factors and Ergonomics Society Annual Meeting, 2016, 60(1): 1823 - 1827.

[25] N. A. Stanton, P. M. Salmon, G. H. Walker, et al. State-ofscience: situation awareness in individuals, teams and systems. Ergonomics, 2017, 60(4): 1-33.

[26] T. Price, M. Tenan, J. Head, et al. Acute stress causes over confidence in Situation Awareness. Proc. of the IEEE International Multi-Disciplinary Conference on the Cognitive Methods in Situation Awareness and Decision Support, 2016: 1-6.

[27] L. R. Marusich, J. Z. Bakdash, E. Onal, et al. Effects of information availability on command-and-control decision making: performance, trust, and situation awareness. Human Factors, 2016, 58(2): $301-321$.

[28] M. R. Endsley, R. P. Smith. Attention distribution and decision making in tactical air combat. Human Factors, 1996, 38(2): $232-249$.

[29] M. R. Endsley. Measurement of situation awareness in dynamic systems. Human Factors, 1995, 37(1): 65-84.

[30] K. Smith, P. A. Hancock. Situation awareness is adaptive, externally directed consciousness. Human Factors, 1995, 37(1): $137-148$.

[31] G. Z. Bedny, M. H. Seglin, D. Meister. Activity theory: history, research and application. Theoretical Issues in Ergonomics Science, 2000, 1(2): 168-206.

[32] M. R. Endsley. Towards a theory of situation awareness in dynamic systems. Human Factors, 1995, 37(2): 32-64.

[33] D. G. Zhang, H. Meng, J. Y. Tao. A method to measure the amount of battlefield satiation information. Proc. of the 19th International Command and Control Research and Technology Symposium, 2014: $1-11$.

[34] H. Meng, D. G. Zhang. Model of situation awareness velocity under continuous changing situation. Systems Engineering and Electronics, 2009, 31(12): 2909-2913. (in Chinese)

[35] E. A. Lee, P. Varaiya. Structure and interpretation of signals and systems. 2nd ed. Boston: Addison-Wesley, 2003.

\section{Biographies}

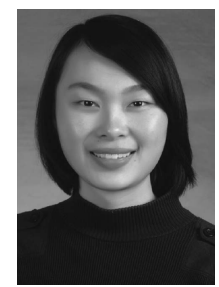

Huiyun Zhao was born in 1990. She is a doctoral candidate in the Army Engineering University, China. Her research interests are situation awareness, military command and control system and complex dynamic network system.

E-mail: huiyun_zhao1819@163.com

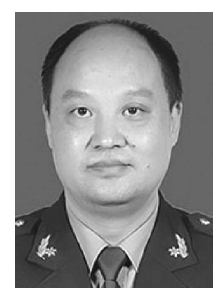

Dongge Zhang was born in 1965 . He is a professor in the Army Engineering University, China. His research interests are analysis of military complex systems, situation awareness, game theory and information economics.

E-mail: DongGeZhang@Hotmail.com

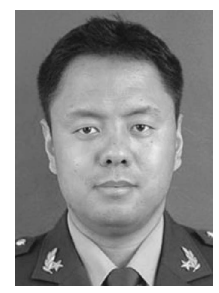

Hui Meng was born in 1981. He is a doctoral candidate in Shanxi University. His research interests are situation awareness and philosophy of science. E-mail: menghui032200@126.com

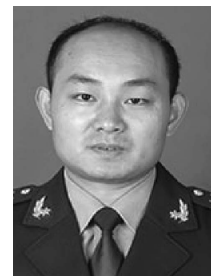

Jiuyang Tao was born in 1983. He is a doctoral candidate in Department of Information Operation \& Command Training, National Defense University, China. His research interests are situation awareness, war gaming, military operation research and intelligent decision-making.

E-mail: taojiuyang@126.com 